\title{
Effect of Viscum album Extract on Angiogenesis Mediators and Cytokines in Egyptian Patients with Intermediate Hepatocellular Carcinoma
}

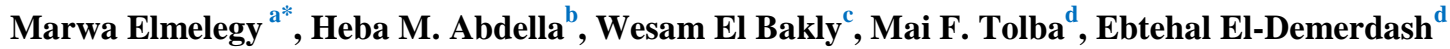 \\ ${ }^{a}$ Department of Clinical Pharmacy, Ain Shams University Specialized Hospital, Cairo 11588, Egypt \\ ${ }^{\mathrm{b}}$ Department of Tropical Medicine, Faculty of Medicine, Ain Shams University, Cairo 11566, Egypt \\ ${ }^{\mathrm{c}}$ Department of Pharmacology and Therapeutics, Faculty of Medicine, Ain Shams University, Cairo 11566, Egypt \\ ${ }^{\mathrm{d}}$ Department of Pharmacology and Toxicology, Faculty of Pharmacy, Ain Shams University, Cairo 11566, Egypt
}

\begin{abstract}
Transarterial chemoembolization (TACE) is the most widely used treatment worldwide for intermediate unresectable hepatocellular carcinoma (HCC). New systemic therapies have shown survival benefits but looking ahead to the future of systemic therapies is necessary to overcome low response rates, relatively high toxicity. The present study aimed to assess the efficacy\& safety of Viscum album in Egyptian patients with intermediate Barcelona Clinic Liver Cancer (BCLC) stage HCC compared to TACE. Forty-five naïve intermediate HCC patients were enrolled in this prospective open-label study. Patients were randomly assigned into 3 groups, Viscum Group I, TACE/Viscum Group II, and TACE Group III. Viscum has been prepared as an injectable aqueous solution containing one milliliter of Viscum. Two Viscum ampoules per week were subcutaneously administered for 8 weeks. All patients underwent laboratory investigations including liver function tests to assess deterioration in liver functions and triphasic spiral computed tomography to assess radiological response according to modified response evaluation criteria in solid tumors (mRECIST). In all cases, chronic hepatitis C virus infection was the cause of liver disease. Stable disease was achieved in all patients. Toxicity, primarily in the form of local reaction and fever, was generally mild \&well tolerated. No discontinuation or toxic deaths associated with drugs were observed. In HCC patients, serum levels of vascular endothelial cell growth factor (VEGF) and Tumor necrosis factor-alpha (TNF- $\alpha$ ) were significantly elevated, whereas levels of Transforming growth factor-B (TGF- $\beta$ ) were lower compared to pretreatment values. In conclusion, in patients with intermediate HCC, Viscum Album is a secure treatment. For efficacy elucidation, further randomized controlled trials with large numbers of patients are recommended. Treatment with TACE and Viscum is associated with modulation of HCC patients with serum angiogenic, inflammatory cytokines.
\end{abstract}

Keywords: hepatocellular carcinoma; Viscum Album; TACE; VEGF; TNF- $\alpha$; TGF- $\beta$.

*Correspondence | Marwa Elmelegy; Department of Clinical Pharmacy, Ain Shams University Specialized Hospital, Cairo 11588, Egypt. Email: marwaabdelsalam333@yahoo.com

Citation | Elmelegy M, Abdella HM, El Bakly W, Tolba MF, El-Demerdash E, 2021. Effect of Viscum album extract on angiogenesis mediators and cytokines in Egyptian patients with intermediate hepatocellular carcinoma. Arch Pharm Sci ASU 5(1): 33-45

DOI: $10.21608 /$ aps.2021.59403.1052

Print ISSN: 2356-8380. Online ISSN: 2356-8399.

Received 09 February 2021. Accepted 5 April 2021.

Copyright: ${ }^{\circledR} 2021$ Elmelegy et al. This is an open-access article licensed under a Creative Commons Attribution 4.0 International License (CC BY 4.0), which permits unrestricted use, distribution, and reproduction in any medium, provided the original author(s) and source are credited. Published by: Ain Shams University, Faculty of Pharmacy

\section{INTRODUCTION}

Hepatocellular carcinoma (HCC) is an aggressive tumor, considered the world's third most prevalent malignancy with a very poor prognosis, making it the fourth leading cause of cancer-related deaths [1]. Hepatitis B or C virus infection, alcohol-induced liver disease, nonalcoholic fatty liver disease, primary biliary cirrhosis, exposure to environmental carcinogens 
such as aflatoxin, and genetic metabolic disorders are common risk factors for HCC [2]. Less than $30 \%$ of patients are eligible for therapies such as transplantation, resection, or ablation that are potentially curative. According to international guidelines, transarterial chemoembolization (TACE) is recommended for selected patients who are not appropriate for such interventions but have the liver-confined disease, preserved liver function, and good performance status [3]. The role of chemotherapy in HCC remains contentious. Unfortunately, a single agent's activity is limited, with only a few drugs showing a response rate of $>10 \%$. In addition, combination chemotherapy has proven equally disappointing because compared to a single agent; additional drugs have resulted in increased toxicity without any increased efficacy. In the same line, the results of clinical studies investigating the efficacy of treatment combinations of antiangiogenic molecules with immune checkpoint inhibitors (ICIs) although encouraging in some cases, but do not fully clarify the best dose, scheduling, and efficacy of these treatments [4]. In the management of HCC patients, developing effective treatment modalities is crucial. TACE is still one of the common modalities in the treatment of patients with unresectable intermediate-stage hepatocellular carcinoma. However, residual viable $\mathrm{HCC}$ is not uncommon following TACE, leading to poor overall survival after TACE alone [5].

Consequently, for these groups of patients, no drug or protocol therapy can be recommended as standard therapy. There is a pressing need to investigate new drugs for these reasons. Viscum album (Mistletoe) is a semi-parasitic plant growing on different host trees. Mistletoe extract has been widely used for several decades as complementary medicine for the treatment of cancer patients and has proven to be beneficial in integrative oncology [6].
Mistletoe therapy in German-speaking countries is one of the most frequently prescribed oncology treatments. As part of integrative oncology (IO) concepts, complementary therapies have been defined and evidence-graded in the guideline of the International Society of Integrative Oncology (SIO) and internationally recognized in the 2018 SIO guideline of the American Society of Clinical Oncology, which considered mistletoe (grade C) to improve the quality of life during and after treatment for breast cancer. Nevertheless, evidence-based guidelines on the use of complementary therapies in Germany for doctors and health professionals are missing [7].

Many biologically active substances comprise the extracted Viscum. As a combined cytotoxic and biological response modifying activity resulting from the activity of plant lectins and other biologically relevant substances, the principle of mistletoe phytotherapeutics may be considered [8]. Twenty-three studies have identified statistically significant positive results for survival, tumor remission, and overall quality of life in a systematic review of controlled clinical trials [9]. Viscum album has shown positive results in advanced HCC and maybe a good opportunity to treat patients with advanced HCC [10]. Traditionally, it is also well known to be an antidiabetic agent. This was done by improving the secretion of insulin and/or improving the action of insulin [11].

In human HCC specimens, vascular endothelial cell growth factor (VEGF), an angiogenic factor involved in neovascularization, is significantly upregulated. Angiogenesis can be induced at the early stages of tumor formation and carcinogenesis [12] and gradually increased during the murine hepato-carcinogenesis procedure; supported by the fact that hepatocarcinogenesis was significantly attenuated by the suppression of the VEGF signaling 
pathway [13]. In addition, hepatic microcirculation is altered at an early stage of liver carcinogenesis, which occurs before the emergence of morphologically identifiable HCC in association with hepatic cellular changes [14].

One component of the immune system, Cytokines, molds the host's response to foreign pathogens and regulates immune cell growth, differentiation, and activation. They are released, including tumors, in response to a different cellular stressor. Th1 cytokines are considered proinflammatory, such as IL-1, IL-2, TNF-alpha, and IFN- $\gamma$, but Th2 cytokines, such as IL-4 and IL-10, are considered anti-inflammatory. Different cytokine receptors are expressed in hepatocytes, such as IL-1 and TNF- $\alpha$. Notably, not only do nonparenchymal cells, such as liver macrophages, synthesize numerous cytokines, but cytokine effects can affect their activity. Liver sinusoidal endothelial cells, in addition, are also the targets and producers of different cytokines. Cytokines have therefore been involved in the pathogenesis of diseases associated with the liver, such as fibrosis, cirrhosis, and cancer [15].

In human cancers, transforming growth factor-beta (TGF- $\beta$ ) plays a dual role because it acts as a tumor suppressor and as a metastasis promoter. In normal cells and early carcinomas, TGF- $\beta$ tumor-suppressive effects, which include inhibition of cell proliferation and apoptosis induction, are observed. On the other hand, in aggressive and invasive tumors, TGF- $\beta$ tumorpromoting effects, including cell adhesion induction, migration, and tumor metastasis, are more clearly observed [16]. Transforming Growth Factor- $\beta$ (TGF- $\beta$ ) is a pleiotropic cytokine that plays a critical role in different cellular process networks, and in recent years, TGF- $\beta$ signaling is involved in tumor initiation and HCC development [17].
Consequently, this study will evaluate the efficacy and safety of Viscum album versus traditional TACE in chemotherapy-naïve intermediate hepatocellular carcinoma in Egyptian patients to further elucidate the possible mechanism of the suppression of hepatocellular carcinoma by Viscum album.

\section{PATIENT AND METHODS}

\subsection{Patients}

This is an open-label, prospective, postmarketing study on the long-term safety and efficacy of Viscum album compared with TACE in intermediate HCC patients. All patients gave written informed consent before enrollment. Before recruitment, the study was approved by the Ain Shams University Faculty of Medicine's ethical review board [Research Ethics committee, Faculty of Pharmacy, Ain Shams University. Master Number: 71]. The study was performed at the HCC unit of Ain Shams University outpatient clinic.

\subsection{Patient characteristics}

All patients fulfilling the inclusion criteria presented to Ain Shams HCC outpatient clinic over 1 year were enrolled in this prospective interventional study. All patients with HCC were diagnosed based on American Association for the Study of Liver Diseases (AASLD) guidelines 2010, Child class A was included in the study. Patients with previous treatment for $\mathrm{HCC}$, advanced hepatic decompensation (Child-Pugh class C); advanced medical co-morbidity; prior systemic therapy; additional malignancy or concomitant anti-tumor therapy including tamoxifen and interferon were excluded from the study.

The study was conducted in the period between March 2016 and February 2017 at El Demerdash Hospital. The study started with 45 patients. They were 28 males and 17 females, 
aged 49 to 64 years with a median of 57 years. Five patients died (one in the first week, two in the fourth week, and two in the last week). Communication was lost with 4 patients, failure to receive the post-treatment samples in 8 patients, and failure to receive post-treatment C.T. in seven patients. At the time of analysis, $21(42 \%)$ patients remained alive with slowly progressive disease (Fig. 1).
Twenty-one patients completed the trial. They were 14 males and 7 females, aged 49 to 64 years. WHO performance status was 3 in all patients. HCV was positive in all patients. Owing to chronic HCV infection, all had liver cirrhosis. All patients were chemotherapy-naive. The median duration of treatment on the Viscum album is 8 weeks. Main portal vein thrombosis was not detected at all and ascites were present in 9 patients (43\%) (Table 1).

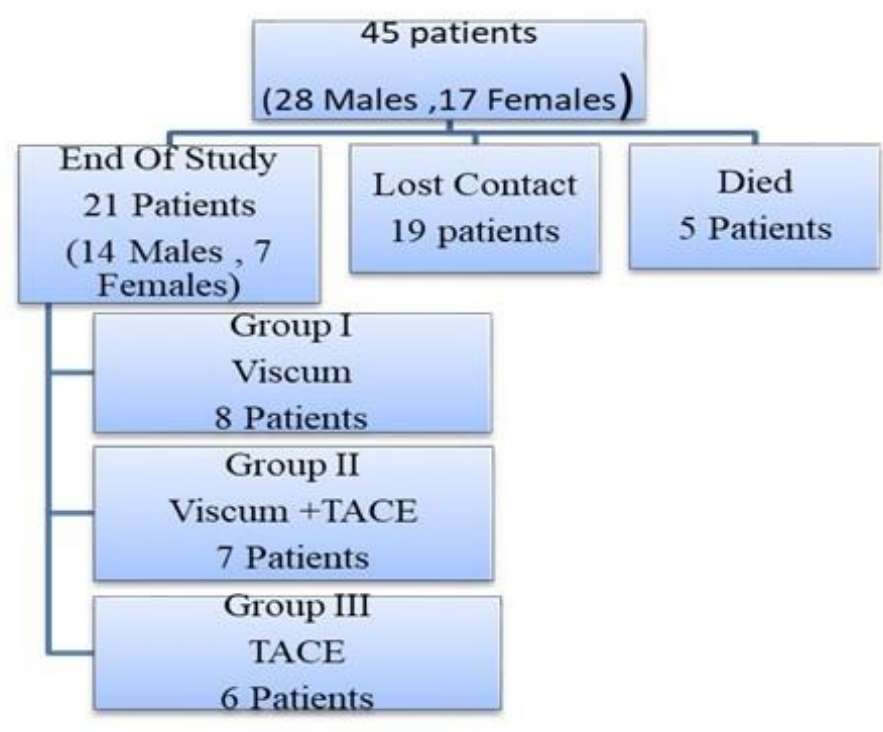

Fig. 1. Flowchart of patient selection

Table 1. Patients Characteristics

\begin{tabular}{lll}
\hline Characteristic & Number of patient & $\%$ \\
\hline $\begin{array}{l}\text { Male: female (total) } \\
\text { Median age in years (range) }\end{array}$ & $14: 7$ & $66 \%$ male \\
$\begin{array}{l}\text { Performance status } \\
\mathbf{1}\end{array}$ & $57(49$ to 64$)$ & \\
$\mathbf{2}$ & 0 & \\
$\mathbf{3}$ & 0 & $100 \%$ \\
Hepatitis & 21 & $100 \%$ \\
HCV (+) & 21 & $100 \%$ \\
Diagnosis: & 21 & \\
Tumor staprotein and imaging & & 0 \\
Main Portal vein Thrombosis & 0 & $43 \%$ \\
Ascites & 9 & \\
\hline
\end{tabular}




\subsection{Methods}

Complete history taking, clinical examination, and laboratory investigations including complete blood picture $(\mathrm{CBC})$, liver enzymes, liver function tests, kidney function tests, $\alpha$-fetoprotein (AFP), and abdominal triphasic spiral CT

TACE procedures were performed by the interventional radiologist through the femoral artery approach in all patients. TACE protocol consisted of intra-arterial infusion of cisplatin 50-100 mg mixed with lipiodoluntil, guided by fluoroscopy to complete filling of the tumor vascular bed and stop-flow in feeding arteries. The number of TACE sessions was decided individually according to patients' follow-up.

Viscum album was an aqueous injectable solution (ATOS Pharma company), with subcutaneous administration of two ampoules once a week.

Forty-five HCC BCLC stage B patients were included in the study. The patients were randomly assigned into 3 groups, with 15 patients in each group. Group I: Viscum album-treated intermediate stage $\mathrm{HCC}$ patients. Group II: TACE and Viscum treated Intermediate stage HCC patients. Group III: TACE treated intermediate stage $\mathrm{HCC}$ patients.

\subsection{Post-treatment follow up}

All patients provided a complete history and physical examination before entry into the study and after 8 weeks of therapy including performance status, concurrent nonmalignant diseases, and therapy. A complete blood cell count, differential count, biochemical liver and renal function tests, electrolyte, chest $\mathrm{x}$-rays, alpha-fetoprotein, computed triphasic liver scan (CT)

\subsection{Response evaluation}

Responses were assessed and verified by C.T. after 8 weeks by independent staff radiologists.
Tumor response has been classified in solid tumor guidelines based on the response evaluation criteria (18). Complete response (CR): the disappearance of any intratumoral arterial enhancement in all target lesions. Partial response (PR): at least $30 \%$ decrease in the sum of diameters of viable tissue. Stable disease (SD) in all other cases. Progressive disease (PD): when there is at least a $20 \%$ increase in the sum of the diameters of viable or enhancing target lesions or if new lesions appear. Disease control $=\mathrm{CR}+\mathrm{PR}$ $+\mathrm{SD}$.

\subsection{Outcome measures}

At the end of the study, the following parameters were measured to the studied groups: C.T., $\alpha$-fetoprotein, VEGF (Vascular endothelial growth factor), TGF- $\beta$ (Transforming growth factor-B (prometastatic), TNF- $\alpha$ (Tumour necrosis factor-alpha). Toxicity was assessed by measuring $\mathrm{CBC}$, Liver function tests, serum creatinine, and fever. All biochemical analyses were done in El Demerdash Hospital as a part of routine work. The detection of serum fetoprotein, TNF- $\alpha$, TGF-B, and VEGF was done by ELISA technique using BioCheck, Inc, Fine Test kit cat no (EH0302; EH0287 and EH0327) respectively.

\subsection{Statistical analyses}

All information is expressed as the mean \pm standard error of the mean (SEM). Differences between groups were assessed using one-way ANOVA followed by multiple comparison tests for post-hoc Tukey-Kramer, or two-way ANOVA repeat measurements followed by posthoc Bonferroni tests. The meaning level $\alpha$ was established at 0.05. Using GraphPad Prism version 5 (GraphPad Software Inc., San Diego, CA, USA), all statistical analyses were performed.

\subsection{Response}

No patients in all groups achieved a complete response according to conventional radiological response criteria (Table 2). 
Table 2. C.T. of HCC patients treated with viscum album (group I), viscum/TACE combined treatment (group II) and TACE (group III)

Group I $\quad$ Group II $\quad$ Group III

Median (Range) Median (Range) Median (Range)

Before treatment

Multiple, $5.1 \mathrm{~cm}(1.5,4.7) \quad$ Multiple, $5 \mathrm{~cm}(3,7)$

Multiple, $5.5 \mathrm{~cm}(3,3.3)$

After treatment

Multiple, $6.9(2.1,5.1)$

Well ablated

Well ablated

Data are presented as median (Range)

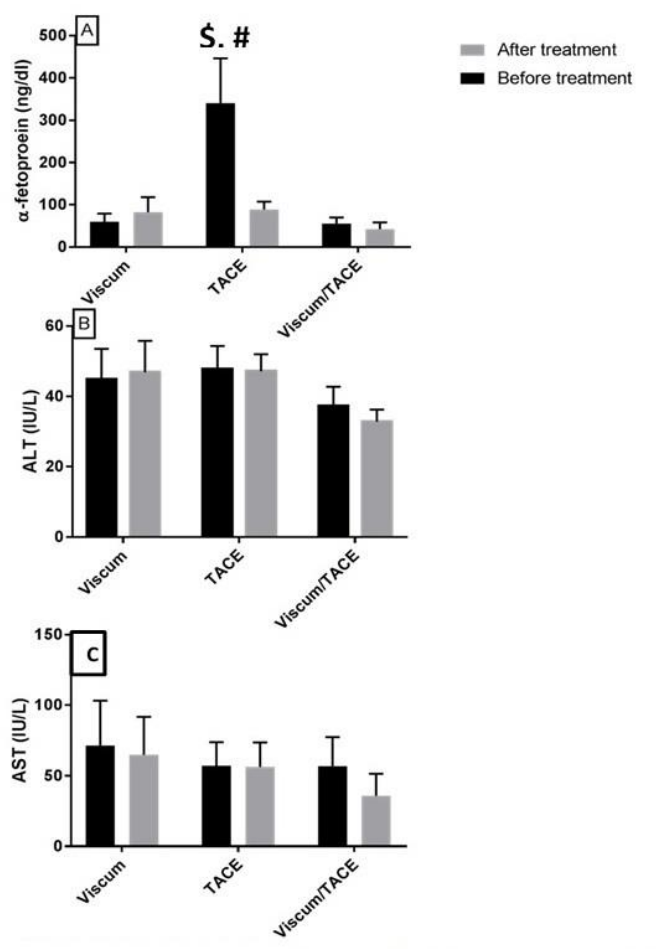

Fig. 2. Serum $\alpha$ - fetoprotein (A), serum ALT (B) and serum AST (C) of HCC patients treated with viscum album, viscum/TACE combined treatment and TACE. Data are presented as mean \pm SEM. analyzed with Two way ANNOVA. a, statistically significant differences between before and after treatment. b, statistically significant differences from viscum. c, statistically significant differences from TACE

\section{RESULTS}

\subsection{Effect of treatment with Viscum album and/or TACE on serum alpha-fetoprotein and liver enzymes.}

Fig. 2 showed the effect of treatment with Viscum and/or TACE on liver enzymes and $\alpha$ - fetoproteins. Viscum album either alone or combined with TACE did not show any significant changes in $\alpha$-fetoproteins, ALT, and AST. However, the baseline alpha-fetoprotein levels were different, it was significantly higher in the TACE group as compared to the other groups (>200 ng/mL) Therefore, AFP change to 
predict the prognosis after may be impossible in this study. Although AFP change after therapies has been proved to correlate the survival of primary HCC patients. TACE treatment alone also showed non-statistically significant changes in liver enzymes after 8 weeks of treatment.

\subsection{Toxicity}

For toxicity, all patients were assessed. Fever associated with drugs has developed in all patients. Five patients died, one in the first week, two in the fourth, and two in the last week. Due to adverse effects such as erythema or injection site pain, seven patients who were excluded from the study reduced the dose to one ampoule over subsequent treatment courses.

\subsection{Effect of treatment with Viscum album and/or TACE on CBC}

Fig. 3 and 4 showed the effect of treatment with Viscum and/or TACE on CBC parameters. Viscum album either alone or combined with TACE did not show any significant changes in all CBC parameters. The only observed insignificant increase in hemoglobin content is mostly due to baseline variability.

\subsection{Effect of treatment with Viscum album and/or TACE on Liver functional test (serum albumin, total bilirubin, direct bilirubin, and INR)}

Fig. 5 showed the effect of treatment with Viscum and/or TACE on serum albumin, total bilirubin, direct bilirubin, and INR. Viscum album either alone or combined with TACE did not show any significant changes in all these parameters. However, combined treatment significantly increased serum albumin content after 8 weeks of treatment by $19 \%$ as compared to the Viscum album.
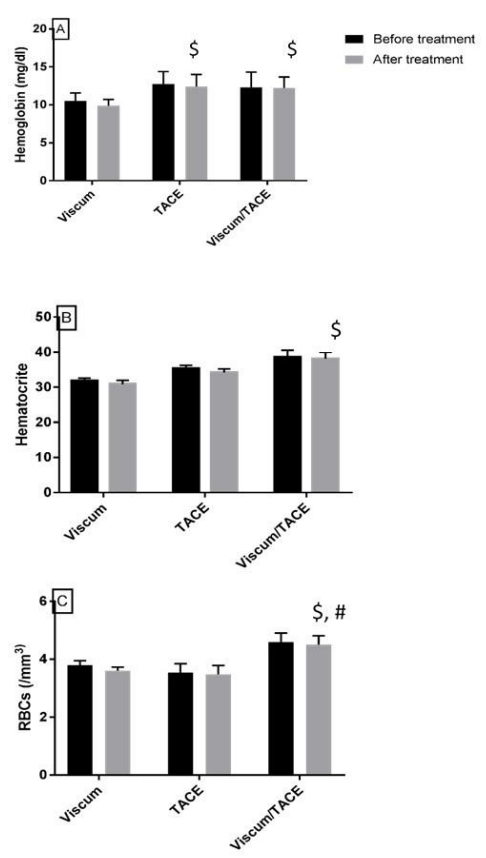

Fig.3. Hemoglobin concentration (A), hematocrit value (B) and RBC (C) of HCC patients treated with viscum album, viscum/TACE combined treatment and TACE. Data are presented as mean \pm SEM. analyzed with Two way ANNOVA. a, statistically significant differences between before and after treatment. b, statistically significant differences from viscum. c, statistically significant differences from TACE
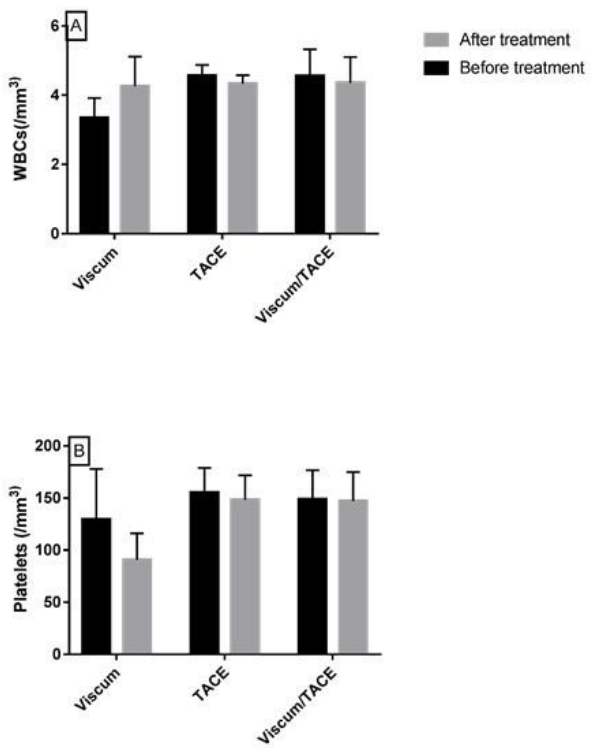

Fig.4. WBC (A), and platelet count (B) of HCC patients treated with viscum album, viscum/TACE combined treatment and TACE. Data are presented as mean \pm SEM. analyzed with Two way ANNOVA 

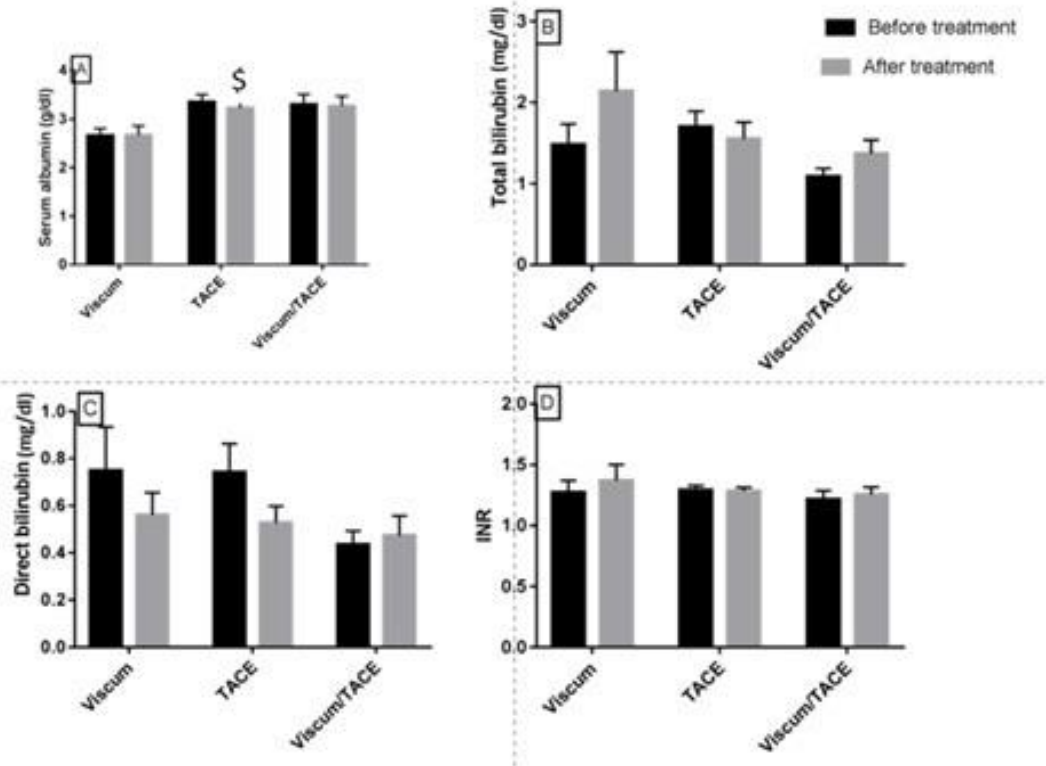

Fig.5. Serum albumin (A), total bilirubin (B), direct bilirubin (C) and INR (D) of HCC patients treated with viscum album, viscum/TACE combined treatment and TACE. Data are presented as mean \pm SEM. analyzed with Two way ANNOVA. b, statistically significant differences from viscum

\subsection{Effect of treatment with Viscum album and/or TACE on serum creatinine}

Fig. 6 showed the effect of treatment with Viscum and/or TACE on serum creatinine. Viscum album either alone or combined with TACE did not show any significant changes in serum creatinine.

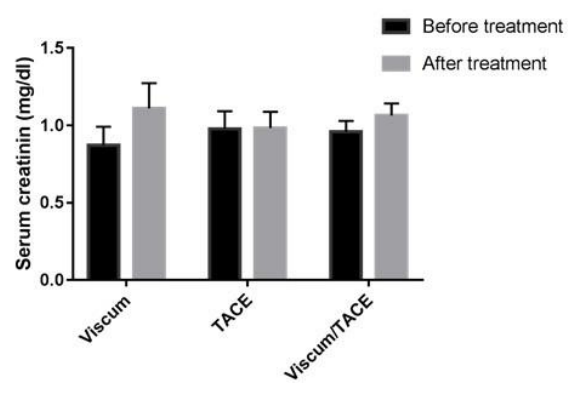

Fig.6. Serum creatinin of HCC patients treated with viscum album, viscum/TACE combined treatment and TACE

\subsection{Survival}

The study began with 45 patients. Five patients died (one in the first week, two in the fourth week, and two in the last week). Communication with 4 patients was lost, 8 patients failed to receive post-treatment samples, and the dose was reduced by seven patients. At the time of the analysis, $21(42 \%)$ patients with slowly progressive disease remained alive.

\subsection{Effect of treatment with Viscum album and/or TACE on serum TNF $\alpha$, VEGF, TGF $\beta$}

Fig. 7 showed the effect of treatment with Viscum and/or TACE on serum TNF- $\alpha$, VEGF, TGF- $\beta$. In naïve patients before treatment, the baseline serum level of TNF $\alpha$ was nonstatistically different between groups. TNF $\alpha$ statistically increased after 8 weeks of treatment with Viscum, Viscum/TACE, and TACE alone by 29,44 , and $22 \%$ respectively, however, there were no statistical differences between different treated groups.

The baseline serum level of VEGF in naive patients before treatment was non-statistically different between the groups. VEGF was statistically increased by $62 \%$ and $98 \%$, respectively, after 8 weeks of treatment with the group I Viscum and group II Viscum/TACE. 
In naïve patients before treatment, the were statistically $(\mathrm{P}<0.05)$ decreased by $9.9 \%$ baseline serum level of TGF- $\beta$ was nonstatistically different between groups. TGF- $\beta$

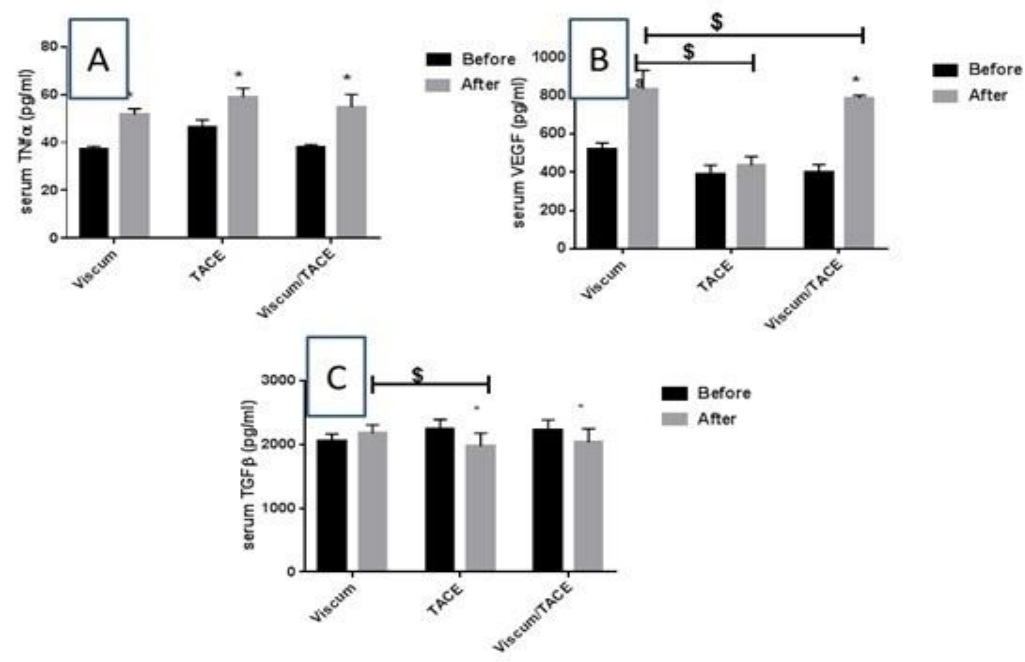

Fig.7. Serum TNF alpha (A), VEGF (B), and TGF- $\beta$ (C) before and after treatment with viscum album, viscum/TACE combined treatment and TACE. in patients with hepatocellular carcinoma. Data are presented as mean \pm SEM. analyzed with Two way ANNOVA. a, statistically significant differences between before and after treatment. b, statistically significant differences from viscum. c, statistically significant differences from TACE

\section{DISCUSSION}

$\mathrm{HCC}$ is a heterogeneous malignancy that is complex to manage, as both the stage of the tumor and any underlying liver disease must be considered jointly [19]. In very advanced cancer stages, it is debatable whether to use conventional therapy that prolongs life with a low probability of success. Although second-line lifeprolonging treatments are now available, no more than the best supportive care is often given to patients, because these treatments can have significant side effects [20].

The efficacy and safety of Viscum album in the treatment of naïve patients with intermediate HCC have been evaluated in the present study. The Viscum album is made with the highest lectin content in aqueous extract. The study was carried out at the HCC unit of Ain Shams University's outpatient clinic. Patients were selected and classified according to the BCLC staging system; the diagnosis was based on guidelines for EASL practice [21]. Patients were divided into 3 groups and received Viscum and/or TACE treatment. Then, VEGF, TGF- $\beta$, and TNF-alpha were evaluated after 8 weeks of treatment to elucidate the possible mechanism of Viscum album to suppress hepatocellular carcinoma. To our knowledge, this is the first research in intermediate HCC to compare and combine the Viscum album with TACE.

The present study was an open (unblinded) trial. In confirmatory clinical trials, non-blinding of the study treatment is always a concern. However, in cancer studies with overall survival as a primary endpoint, it is not essential according to FDA guidance blinding. In addition, the CHOCRANE review [22] reported that the efficacy findings did not differ significantly in studies with continuously distributed or binary endpoints, such as quality-of-life data, with the use of blinded (placebo) versus unblinded (open) 
control groups. In addition, treatment with mistletoe usually induces dermal erythema at the injection site; the value of blinding may therefore be questionable.

The findings of the current study showed that none of the patients achieved a complete response in all the groups. Viscum album showed no significant changes in alpha-fetoprotein, ALT, and AST compared to pretreatment values, either alone or in combination with TACE. After 8 weeks of treatment, TACE treatment alone also showed non-statistically significant changes in liver enzymes. Interestingly, partial remission was only seen in patients treated with the Viscum/TACE combination therapy regimen, so it is not possible to rule out the possibility of synergistic antineoplastic effects between Viscum and TACE. However, given the limited sample size, and nonblindness, no conclusions should be drawn regarding the influence of combined treatment on disease response in the current study.

In their controlled, randomized studies of head and neck cancer and non-small cell lung cancer, our results mimic the findings of SteuerVogt, et al. [23], which showed no significant effect of Viscum extract therapy on tumor recurrence and survival. On the other hand, in a systematic review, there are several controlled, prospective studies in which the clinical efficacy of Viscum extract has shown some positive effects on breast and gynecological cancer [24]. In addition, the Viscum album has been nontoxic and offers patients with locally advanced or metastatic pancreatic cancer a prolongation of overall survival as well as fewer disease-related symptoms. In addition, a xenograft mouse model strongly inhibits the proliferation of $\mathrm{HCC}$ cells and tumor tissue primarily by destabilizing the proto-oncogene c-Myc [25]. Concerning toxicity, the treatment with Viscum album extract was well tolerated in the present study, with no serious or life-threatening undesirable effects, in particular no serious allergic reactions. After Viscum album treatment, anemia was apparent and these changes were not statistically significant for pretreatment values. When Group II and Group III were compared with Group I, hemoglobin, hematocrit, and RBCs increased statistically significantly. No effect on the kidney function test in all patients was observed. Collectively, no drug-related toxicity was observed in functional tests of the kidney and liver. In addition, the incidence of systemic drug adverse reactions and local injection site reactions were of the same magnitude and quality as in previously published clinical trials of Viscum album extracts [26]. As a result, complementary treatment with standardized HCC mistletoe extract can be considered to be safe.

There is a wide range of information on the incidence of adverse drug reactions in response to Viscum album therapy and there is ongoing debate as to whether certain reactions are adverse drug reactions or desired immunoreactions. Elevated temperature in Viscum album treatment is a well-known and even desired reaction. According to product information, it is part of the dose-finding strategy in systemic therapy, and mistletoe-induced fever is described as immunostimulation. Limited toxicities included flu-like symptoms, gingivitis, eosinophilia, and a slight increase in serum urea nitrogen and creatinine were reported in a phase I study in HIV-positive patients treated with Viscum album. Mistletoe preparations, therefore, appear to be well-tolerated, and antibody reactions seem to be robust (27). Notably, fewer adverse events and longer survival were observed in the mistletoe therapy group compared to conventional therapy alone in a large retrospective study of mistletoe therapy in nonmetastatic breast cancer patients. Similar clinical results have been observed in a comparative retrospective study design in patients with 
pancreatic cancer, with fewer adverse events and improved survival in patients treated with mistletoe [28].

Finally, we assessed the effect of Viscum and/or TACE treatment on serum TNF- $\alpha$, VEGF, TGF- $\beta$ to elucidate the possible mechanism by which these treatments could affect HCC. In naïve patients before treatment, baseline serum levels of TNF $\alpha$, VEGF, and TGF- $\beta$ were nonstatistically different between groups. TNF $\alpha$ statistically increased after treatment with Viscum, Viscum/TACE, and TACE alone by 29 , 44 , and $22 \%$ respectively, however, there were no statistical differences between different treated groups. VEGF was statistically increased after treatment with Viscum or Viscum/ TACE by $62 \%$ and $98 \%$, respectively. TGF- $\beta$ was statistically decreased by $9.9 \%$ after treatment with TACE only.

The present findings agree with those of Mansky, et al [29]. Extracts of the European Viscum album L. are commonly used in Europe to treat cancer. Several studies have reported immunostimulatory effects on mononuclear cells, lymphocytes, macrophages, and NK cells from mistletoe extracts. After all, there is controversy as to how the plant extracts exert their postulated dual cytotoxicity activity towards tumor cells and immune cell stimulation. Viscum album extract immunostimulatory effects have been assigned to a low molecular weight oligosaccharide compound. In addition, the stimulatory effect on the activity of natural killer (NK) cells appears to result from enhanced production by $\mathrm{T}$ cells and macrophages of interferon (INF- $\gamma$ ) and tumor necrosis factor- $\alpha$ (TNF- $\alpha$ ). On the other hand, there is some evidence that in mistletoe preparations, lectin components are important effector molecules. The components of lectin increase the total number and activity of neutrophils, NK cells, and large granular lymphocytes and activate subpopulations of monocyte-macrophages and helper cells simultaneously. Higher levels of cytokines such as interleukin IL-1, IL-6, granulocytemacrophage colony-stimulating factor (GMCSF), and TNF-a were associated with this [30].

Viscum extracts have been reported to exert an antiangiogenic effect, in addition to the immunostimulatory effect of mistletoe extract and lectins [31]. A wide range of phenomena, including increased division of endothelial cells within the tumor and up-regulation of cell adhesion molecules, are the mechanisms involved in tumor angiogenesis. Viscum preparation has been shown to induce dose- and time-dependent apoptosis of immortalized endothelial cells. Viscum extracts also inhibit the growth of tumors and endothelial cells by delaying the progression of cell cycles and causing apoptotic cell death [32].

To sum up, complementary and alternative medicine (CAM) continues to present health care professionals with a challenge. Since CAM use is increasing in cancer patients, it is important to ensure that use is adequate to minimize unfavorable adverse effects between CAM and conventional cancer treatment [33].

In conclusion, the results of the current study indicate that the Viscum album can be administered to patients with intermediate HCC because it has virtually no side effects and a low profile of toxicity. Further studies are warranted on the use of the Viscum album in combination with conventional treatment.

\section{Declarations}

\section{Ethics approval and consent to participate}

Not applicable

\section{Consent to publish}

Not applicable

\section{Availability of data and materials}

All data generated or analyzed during this study are included in this published article in the 
main manuscript.

\section{Competing interests}

No competing interests were declared by the authors.

\section{Funding statement}

No funding source was received

\section{REFERENCES}

1. Kole C, Charalampaki N, Tsakatikas S, Vailas M, Moris D, Gkotsis E, et al. Immunotherapy for Hepatocellular Carcinoma: A 2021 Update. Cancers (Basel) $2020 \quad$ Oct 4;12(10):2859. doi: $10.3390 /$ cancers 12102859 .

2. McGlynn A, Petrick Jessica L, and. El-Serag Hashem B. Epidemiology of Hepatocellular Carcinoma. Hepatology, vol. 73, no. S1, 2021: Katherine

3. Cicalese L. Hepatocellular Carcinoma (HCC) Guidelines Updated: Jun 05, 2020, https://emedicine.medscape.com/article/197319guidelines

4. Massena M, Busato D, Baboci L, Di Cintio F, Toffoli G, and Dal Bo M. New Insight into Therapies Targeting Angiogenesis in Hepatocellular Carcinoma. Cancers 2019, 11, 1086; doi:10.3390/cancers 11081086

5. Kudo M. A Paradigm Change in the Treatment Strategy for Hepatocellular Carcinoma. Liver Cancer 2020; 9:367-377 368: www.karger.com/lic DOI: $10.1159 / 0005079342020$

6. Hwang WY, Kang MH, Lee SK, Yeom JS, Jung $\mathrm{MH}$. Prolonged stabilization of platinum-refractory ovarian cancer in a single patient undergoing longterm Mistletoe extract treatment Case report. Medicine (2019) 98:8 (e14536) ttp://dx.doi.org/10.1097/MD.0000000000014536

7. Matthes H, Thronicke A, Hofheinz RD, Baars E, Martin D and Hube R. Statement to an Insufficient Systematic Review on Viscum album L. Therapy. Evidence-Based Complementary and Alternative Medicine Volume 2020, Article ID 7091039, 9 pages https://doi.org/10.1155/2020/7091039

8. Zarkovic N, Vukovic T, Loncaric I, et al. An Overview on Anticancer Activities of the Viscum
Album Extract Isorel (B) 2002. Cancer Biother Radiopharm 16:55-62. doi: $10.1089 / 108497801750096041$

9. Kienle GSK, Kiene H. Complementary cancer therapy: a systematic review of prospective clinical trials on anthroposophic mistletoe extracts. Eur J Med Res 2007 12:103-119.

10. Mabed M, El-Helw L, Shamaa S. Phase II study of Viscum fraxini-2 in patients with advanced hepatocellular carcinoma. Br J Cancer 2004. 90:6569. doi: $10.1038 /$ sj.bjc. 6601463

11. Gray AM, Flatt PR Nature's own pharmacy: The diabetes perspective. Proc Nutr Soc 2008: 56:507517. doi: $10.1079 /$ pns 19970051

12. Han M, Liao Z, Liu F, Chen X, Zhang C, Zhong C, et al. Modulation of the TGF- $\beta$ signaling pathway by long noncoding RNA in hepatocellular carcinoma. Biomarker Research 2020: 8:70 https://doi.org/10.1186/s40364-020-00252-x

13. Yoshiji H, Kuriyama S, Yoshii J, et al. Halting the interaction between vascular endothelial growth factor and its receptors attenuates liver carcinogenesis in mice. Hepatology 2004: 39:15171524. doi: 10.1002/hep.20218

14. Frachon S, Gouysse G, Dumortier J, et al. Endothelial cell marker expression in dysplastic lesions of the liver: An immunohistochemical study. J Hepatol 2001: 34:850-857. doi: 10.1016/S0168-8278(01)00049-6

15. Lingxiao FQI, Huang WP, Zhao Z, Yang B, Xia. Time-series clustering of cytokine expression after transarterial chemoembolization in patients with hepatocellular carcinoma. Oncology Letters 19: 1175-1186, $2020 \quad$ DOI: 10.3892/ol.2019.11209

16. Huang JJ, Blobe GC. Dichotomous roles of TGF- $\beta$ in human cancer. Biochem Soc Trans 2016: 44:1441-1454. doi: 10.1042/bst20160065

17. Galle PR, Forner A, Llovet JM, et al. EASL Clinical Practice Guidelines: Management of hepatocellular carcinoma. J Hepatol 2018: 69:182236.

doi: 10.1016/j.jhep.2018.03.019

18. Therasse P. European Organization for Research and Treatment of Cancer, National Cancer Institute of the United States, National Cancer Institute of 
Canada. J Natl Cancer inst 2000: 92:205-16

19. Rich NE, John BV, Parikh ND, Rowe J, Mehta N, Khatri G, Thomas SM, et al. Hepatocellular Carcinoma Demonstrates Heterogeneous Growth Patterns in a Multicenter Cohort of Patients with Cirrhosis. HEPATOLOGY 2020, VOL. 72, NO. 5: 1654-1565.

onlinelibrary.wiley.com/doi/10.1002/hep.31159/sup pinfo.

20. Tröger W, Galun D, Reif M, et al.Viscum album [L.] extract therapy in patients with locally advanced or metastatic pancreatic cancer: A randomized clinical trial on overall survival. Eur $\mathbf{J}$ Cancer 2013: 49:3788-3797. doi: 10.1016/j.ejca.2013.06.043

21. Mehta, N. Hepatocellular Carcinoma-How to Determine Therapeutic Options. Hepatology Communications 2020;4:342-354 DOI 10.1002/hep4.1481

22. Hróbjartsson A, Gøtzsche PC Placebo interventions for all clinical conditions. Cochrane Database Syst. Rev. 2010

23. Steuer-Vogt M., Bonkowski V, Ambrosch P, et al.The effect of an adjuvant mistletoe treatment program in resected head and neck cancer patients. Eur J Cancer 2002: 37:23-31. doi: 10.1016/s09598049(00)00360-9

24. Kienle GS, Glockmann A, Schink M, Kiene H. Viscum album L. extracts in breast and gynecological cancers: A systematic review of clinical and preclinical research. J Exp Clin Cancer Res. 2009 doi: 10.1186/1756-9966-28-79

25. Ding X, Cartwright C, Tan L, et al: Abstract 3206: Mistletoe extract inhibits the proliferation of human hepatocellular carcinoma cells by induction of apoptosis and downregulation of c-MYC. Cancer Res 2015: 74:3206-3206. doi: 10.1158/15387445.am2014-3206

26. Bock PR, Friedel WE, Hanisch J, et al. Efficiency and safety of long-term complementary treatment with standardized European mistletoe extract (Viscum album L.). ARZNEIMITTELFORSCHUNG-DRUG Res 2004: 54:456-466.

27. Huber R, Eisenbraun J, Miletzki B, et al: Pharmacokinetics of natural mistletoe lectins after subcutaneous injection. Eur J Clin Pharmacol: 2010: 66:889-897. doi: 10.1007/s00228-010-08305

28. Matthes H, Friedel WE, Bock PR, Zanker KS. Molecular Mistletoe Therapy: Friend or Foe in Established Anti-Tumor Protocols? A Multicenter, Controlled, Retrospective PharmacoEpidemiological Study in Pancreas Cancer. Curr Mol Med: 2010: 10:430-439. doi: $10.2174 / 156652410791317057$

29. Mansky P, Wallerstedt DB, Sannes T, Stagl J, Johnson LL, Blackman MR et al. NCCAM/NCI Phase 1 study of Mistletoe Extract and gemcitabine in patients with advanced solid tumors. Evid Based Complement Alternat Med 2013;2013: doi: $10.1155 / 2013 / 964592$

30. Mueller EA, Anderer FA. Synergistic Action of a Plant Rhamnogalacturonan Enhancing Antitumor Cytotoxicity of Human Natural Killer and Lymphokine-activated Killer Cells: Chemical Specificity of Target Cell Recognition. Cancer Res: 1990: 50:3646-3651

31. Park W-B, Lyu S-Y, Kim J-H, et al: Inhibition of Tumor Growth and Metastasis by Korean Mistletoe Lectin is Associated with Apoptosis and Antiangiogenesis. Cancer Biother Radiopharm 2002: 16:439-447. doi: $10.1089 / 108497801753354348$

32. Elluru SR, Van Huyen JPD, Delignat S, et al: Antiangiogenic properties of Viscum album extracts are associated with endothelial cytotoxicity. Anticancer Res 2009: 29:2945-2950

33. Jones E, Nissen L, McCarthy A, Steadman K and Windsor C. Exploring the Use of Complementary and Alternative Medicine in Cancer Patients. Integrative Cancer Therapies Volume 2019: 18: 1-9 (C) DOI: $\quad 10.1177 / 1534735419846986$ journals.sagepub.com/home/ict). 